\title{
Properdin binds independent of complement activation in an in vivo model of anti-glomerular basement membrane disease
}

\section{O'Flynn, Joseph}

2018-12

O'Flynn , J , Kotimaa , J , Faber-Krol , R , Koekkoek , K, Klar-Mohamad, N , Koudijs , A , Schwaeble , W J , Stover , C , Daha, M R \& van Kooten , C 2018 , ' Properdin binds independent of complement activation in an in vivo model of anti-glomerular basement membrane disease ' , Kidney International , vol. 94 , no. 6 , pp. 1141-1150 . https://doi.org/10.1016/j.kint.2018.06.03

http://hdl.handle.net/10138/307703

https://doi.org/10.1016/j.kint.2018.06.030

publishedVersion

Downloaded from Helda, University of Helsinki institutional repository.

This is an electronic reprint of the original article.

This reprint may differ from the original in pagination and typographic detail.

Please cite the original version. 


\section{Properdin binds independent of complement activation in an in vivo model of anti-glomerular basement membrane disease}

Joseph O'Flynn ${ }^{1,4}$, Juha Kotimaa ${ }^{1,2,4}$, Ria Faber-Krol ${ }^{1}$, Karin Koekkoek , Ngaisah Klar-Mohamad ${ }^{1}$, Angela Koudijs ${ }^{1}$, Wilhelm J. Schwaeble ${ }^{3}$, Cordula Stover ${ }^{3}$, Mohamed R. Daha ${ }^{1}$ and Cees van Kooten

${ }^{1}$ Leiden University Medical Center, Department of Nephrology, Leiden, The Netherlands; ${ }^{2}$ University of Helsinki, Faculty of Medicine, Department of Bacteriology and Immunology, Helsinki, Finland; and ${ }^{3}$ University of Leicester, Department of Infection, Immunity and Inflammation, Leicester, UK

Properdin is the only known positive regulator of complement activation by stabilizing the alternative pathway convertase through C3 binding, thus prolonging its half-life. Recent in vitro studies suggest that properdin may act as a specific pattern recognition molecule. To better understand the role of properdin in vivo, we used an experimental model of acute anti-glomerular basement membrane disease with wild-type, C3- and properdin knockout mice. The model exhibited severe proteinuria, acute neutrophil infiltration and activation, classical and alternative pathway activation, and progressive glomerular deposition of properdin, C3 and C9. Although the acute renal injury was likely due to acute neutrophil activation, we found properdin deposition in C3-knockout mice that was not associated with lgG. Thus, properdin may deposit in injured tissues in vivo independent of its main ligand C3. Kidney International (2018) 94, 1141-1150; https://doi.org/10.1016/ j.kint.2018.06.030

KEYWORDS: alternative pathway; C1q; C3; C5b-9; classical pathway; complement; glomerulonephritis; inflammation glomerular basement membrane; properdin

Copyright (c) 2018, International Society of Nephrology. Published by Elsevier Inc. All rights reserved.

Correspondence: Cees van Kooten, LUMC, Department of Nephrology, P.O. Box 9600, 2300 RC Leiden, The Netherlands. E-mail: kooten@lumc.nl

${ }^{4}$ These authors contributed equally to this work.

Received 5 July 2017; revised 31 May 2018; accepted 28 June 2018; published online 12 October 2018 he complement system consists of 3 activation pathways, classical (CP), lectin (LP), and alternative (AP), which converge at the level of $\mathrm{C} 3$ activation, proceeding to $\mathrm{C} 5$ cleavage and initiating the terminal pathway $\mathrm{C} 5-\mathrm{C} 9$ activation. Both the $\mathrm{CP}$ and $\mathrm{LP}$ have pattern recognition molecules (PRM), such as C1q and MBL, which in combination with the associated proteases initiate the complement cascade. ${ }^{1}$ AP has an important role in augmenting $\mathrm{CP}$ and LP; once $\mathrm{C} 3$ is activated, the AP amplification loop enhances the complement cascade activation. ${ }^{2,3}$ In addition, there is a constant low-level auto-activation of C3 (tick-over), which allows C3b formation on surfaces of pathogens and injured host cells. ${ }^{1,4}$ The membrane-bound C3b associates with factor $\mathrm{B}$, forming a short-lived AP C3 convertase $\mathrm{C} 3 \mathrm{bBb}$, which properdin binding stabilizes significantly, and making properdin the only positive regulator of complement. ${ }^{5-8}$

Recent in vitro studies have shown that properdin may exhibit PRM activity. Studies have shown targeted complement-fixing activity on certain ligands such as lipopolysaccharides (LPS), ${ }^{9}$ oxidized LDL, ${ }^{10}$ and myeloperoxidase. ${ }^{11}$ Similar results have been demonstrated with apoptotic and necrotic cells, and heparan sulfates expressed at the surface of renal epithelial cells. ${ }^{12-16}$ In this model properdin attaches to specific ligands and forms stable C3bBbP convertase, possibly overcoming the local threshold for C5 convertase (C3bBbC3b) formation. ${ }^{8,9,12}$ However, it is not yet clear how properdin could act as a PRM, as it lacks known recognition domains. ${ }^{17}$

$\mathrm{AP}$ and properdin have been shown to contribute to arthritis, abdominal aortic aneurysm, and renal ischemia reperfusion injury (I/RI). However, these studies have not been able to differentiate whether the deposition of properdin in tissues takes place via membrane-bound C3b or through epitopes present in the injured tissues. ${ }^{18-21}$ To study the sequence of properdin binding in vivo, we established an acute model of anti-glomerular basement membrane (GBM) disease in wild-type (WT), C3 knockout (KO), and properdin KO mice. Recent studies on anti-GBM disease have shown that properdin and other AP components are prominently present in anti-GBM-affected glomeruli in humans and that AP contributes to the injury in mice, ${ }^{22-24}$ making the model attractive for studying properdin. 


\section{RESULTS}

Characterization of acute complement activation after antiGBM antibody initiation

Acute complement activation was evaluated with comparison of mouse plasma before and 2 hours post-administration of anti-GBM antibody, showing significantly increased C3b/C $3 \mathrm{c} /$ iC3b in WT mice (3.6-fold), which was not seen following administration of control IgG or in properdin-KO (fP KO) mice (Figure 1a). Moreover, basal levels of C3 activation fragments was 2-fold lower in $\mathrm{fP} \mathrm{KO}$ mice compared with the WT mice.

The novel mouse properdin enzyme-linked immunosorbent assay (ELISA) has a linear detection range with recombinant mouse properdin (Figure 1b) with no detectable properdin in $\mathrm{fP} \mathrm{KO}$ mouse serum (Figure 1c). No changes in circulating properdin were found 2 hours after injection of WT mice with anti-GBM or control antibody (Figure 1c).

Functional ELISAs established intact serum complement in WT mice and reduced AP activity in the $\mathrm{fP} \mathrm{KO}$ mice. Two hours after anti-GBM antibody injection, prominent consumption of CP (Figure 1d) and AP (Figure 1e) was seen, whereas findings for LP consumption were inconclusive (Figure 1e). No consistent complement consumption was observed with control antibody-injected mice. In properdin KO mice anti-GBM antibody injection did not result in significant CP or LP pathway consumption (Figure 1f). Together these results show prominent complement activation in this anti-GBM model, with partial dependence on properdin.

\section{Time course histological analysis of complement activation in glomeruli}

The kinetics of renal complement activation following antiGBM antibody injection were evaluated in groups of WT mice killed at different time points. The anti-GBM rabbit IgG (Rb IgG) was clearly present in glomerular vasculature from 2 to 72 hours (Figure 2). The C1q staining was performed as a marker of CP involvement, showing an acute and unchanged staining pattern from 2 to 72 hours, closely resembling that of $\mathrm{Rb}$ IgG (Figure 2). The C3 deposition was present in glomeruli at 2 hours and increased until 72 hours. The C3 deposition closely resembled the pattern of rabbit IgG until 48 hours, after which a focal staining pattern became more apparent (Fig 2). Properdin deposition was present at 2 hours, with clear increase from 24 hours onwards. Interestingly, properdin (fP) staining did not exhibit the linear pattern found with IgG and C3 (Figure 2). The staining for both C6 and C9 exhibited late kinetics, emerging only after 24 hours and becoming most prominent at 48 hours (Figure 2). In all cases specificity of the staining was confirmed with isotype and biological positive and negative controls (Supplementary Figure S1). We did not observe gender-specific differences in staining pattern or intensity in any of the studied complement factors (data not shown).
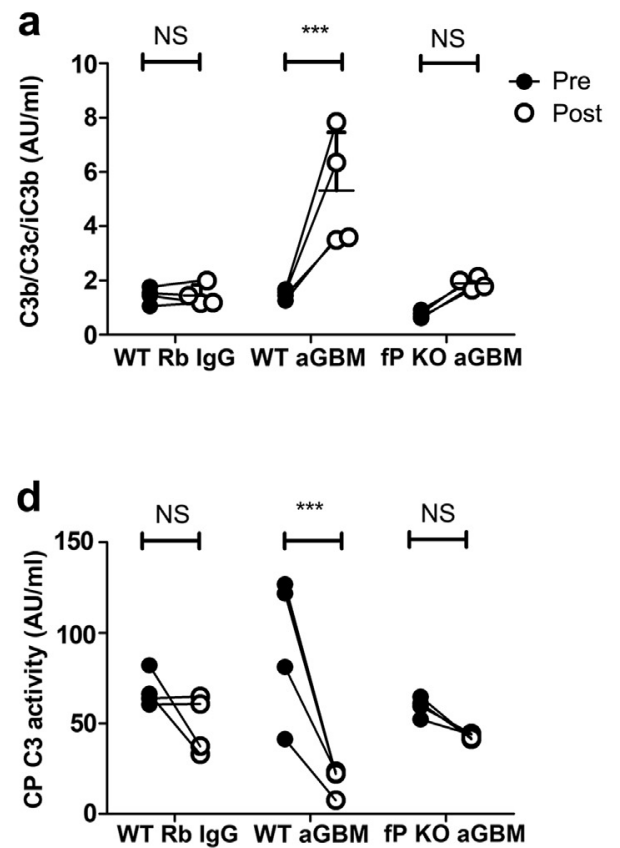
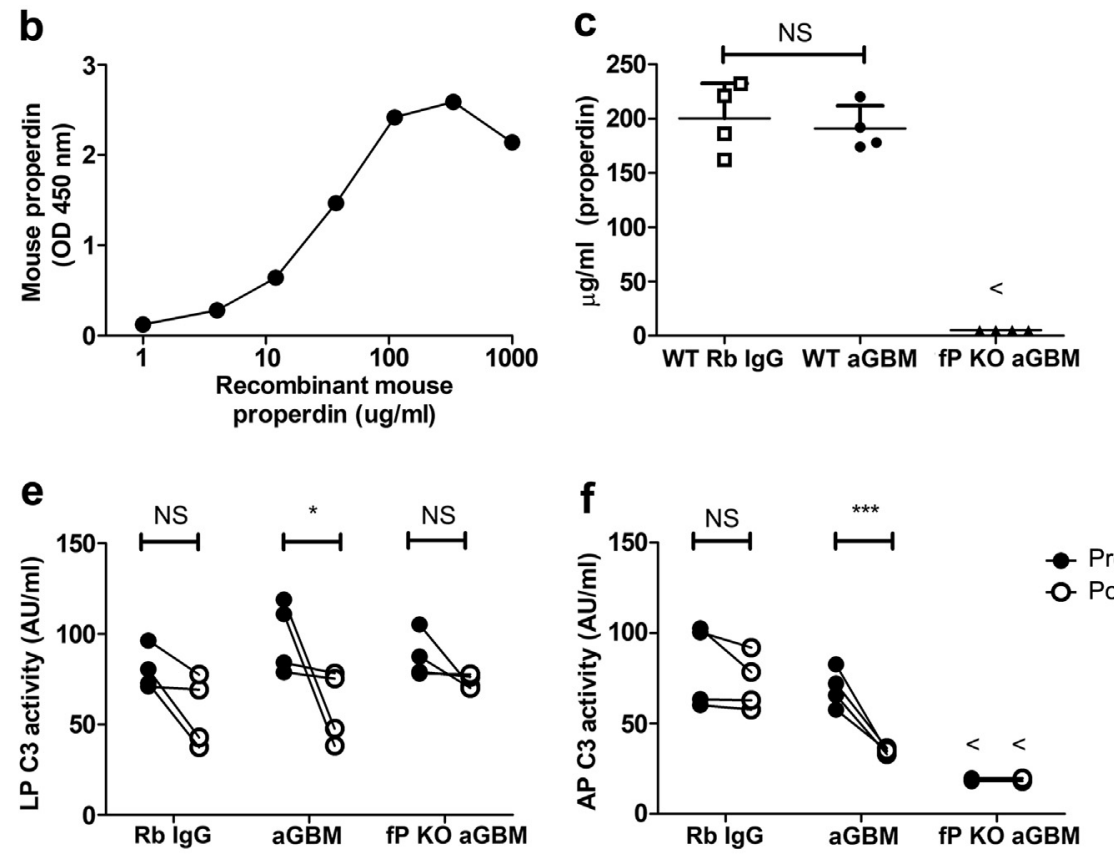

Figure 1 | Analysis of systemic complement. (a) Acute complement activation was determined with plasma C3b/C3c/iC3b at 2 hours with paired pre- and post-injection samples from anti-glomerular basement membrane (GBM) antibody-treated wild-type (WT) and properdin knockout (fP KO) mice and control polyclonal antibody (pAb)-treated WT mice. (b) Properdin sandwich enzyme-linked immunosorbent assay (ELISA) performance was assessed with reciprocal dilutions of recombinant mouse properdin with 4-fold steps from $1000 \mathrm{ng} / \mathrm{ml}$. (c) Serum properdin levels were assessed 2 hours after induction of the model with anti-GBM antibody in WT and fP KO mice, and with control pAbinjected WT mice. (d) Serum classical (CP), (e) lectin (LP), and (f) alternative (AP) pathway activities were determined with functional C3 ELISAs. Two-way analysis of variance (ANOVA) was used to determine change specificity in pre- and post-injection analysis, and 1-way ANOVA was used to determine specificity of change between control and anti-GBM antibodies. Error bars represent SD of duplicate measurements. ${ }^{*} P<0.05$, ${ }^{* * *} P<0.001$. aGBM, anti-GBM antibody; NS, not significant; Rb IgG, rabbit anti-GBM lgG. 
$2 \mathrm{~h}$

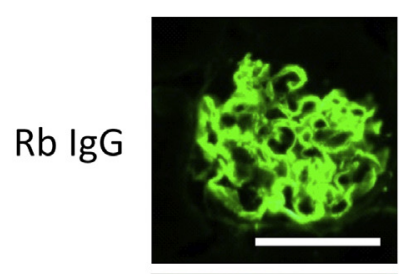

C1q

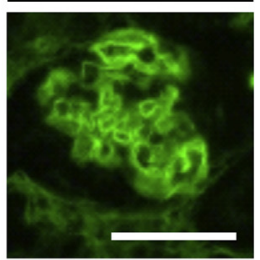

C3

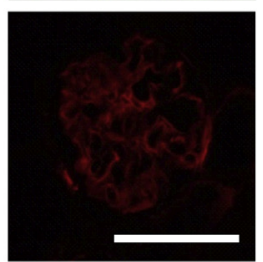

fP

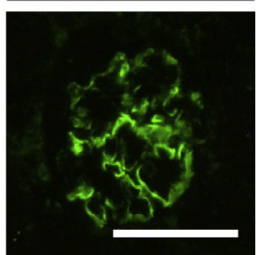

C6

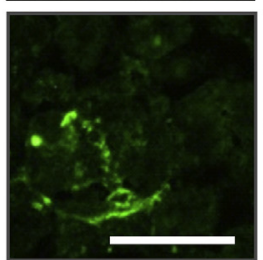

C9

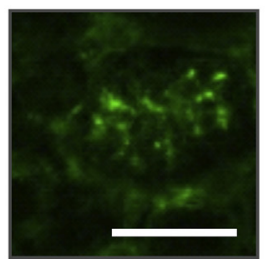

$6 \mathrm{~h}$
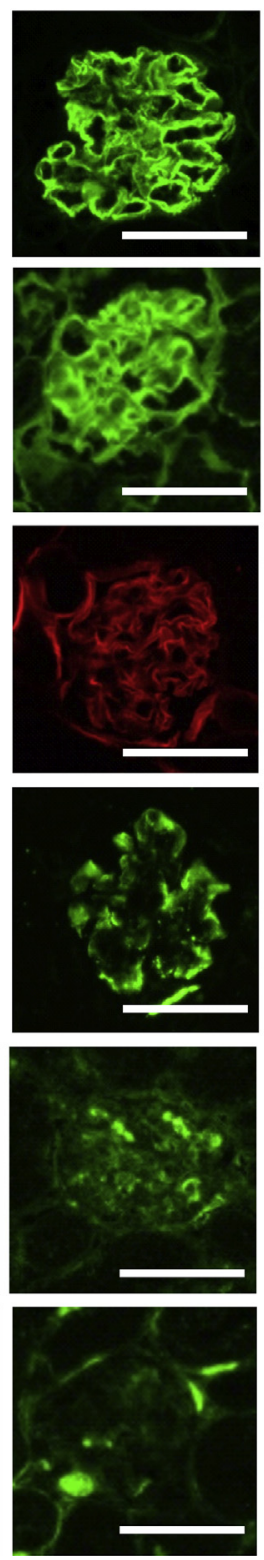

$24 \mathrm{~h}$
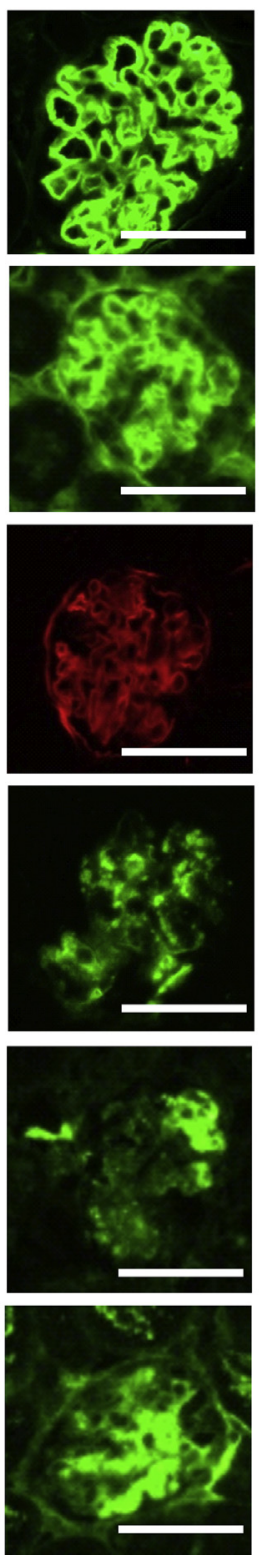

$48 \mathrm{~h}$
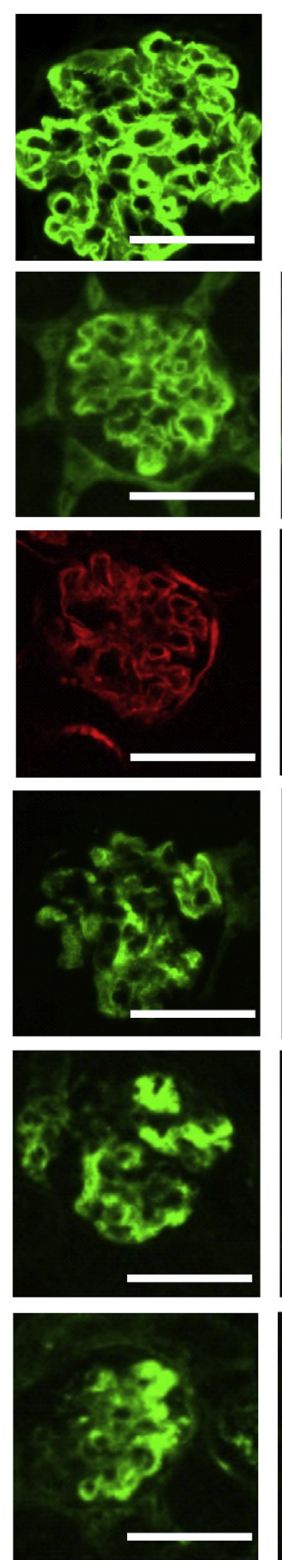

$72 \mathrm{~h}$
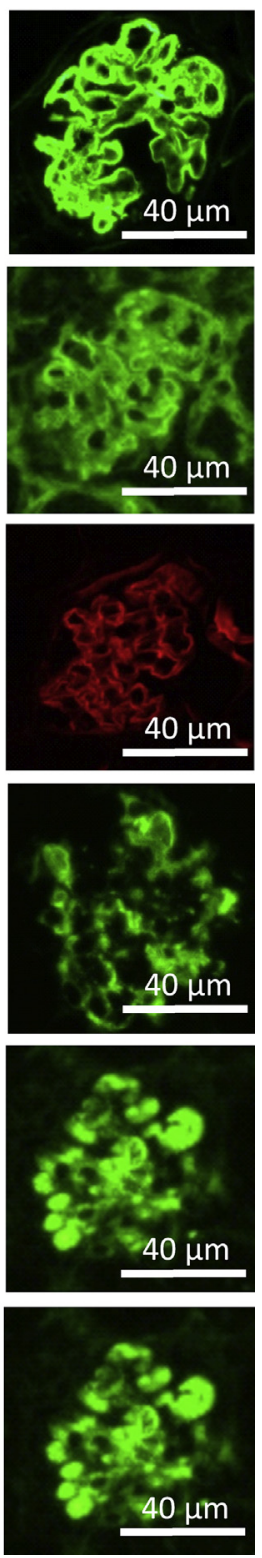

Figure 2 | Time-course analysis of glomerular activation of complement. Analysis was performed with kidneys harvested from wild-type C57bl/6 mice killed 2 to 72 hours ( $h$ ) after administration of anti-glomerular basement membrane (GBM) lgG. Deposition of rabbit anti-GBM lgG (Rb lgG) and mouse C1q (green) were detected with Alexa-488 secondary antibody. C3 deposition (red) was detected with Alexa-568-labeled secondary antibody. Mouse properdin, C6, and C9 deposition (green) was detected with HRP-labeled secondary antibody and tyramide-FITC amplification. Images were acquired at $40 \times$ original magnification, 4 mice ( 2 female and 2 male) per group were analyzed, and the representative glomerulus was chosen from images acquired from 4 different animals. To optimize viewing of this image, please see the online version of this article at www.kidney-international.org.

\section{C3 and properdin depositions show progressive increase and colocalization}

The rapid deposition of $\mathrm{C} 3$ in the glomerulus, combined with the systemic consumption of $\mathrm{CP}$ and linear deposition of both $\mathrm{Rb}$ IgG and $\mathrm{Clq}$, suggested an IgG- and CP-mediated activation mechanism. To verify this, we assessed glomerular colocalization of $\mathrm{Rb}$ IgG and C3 with anti-GBM antibodyinjected WT mice. The time-course histology showed that the deposition of C3 closely resembles both rabbit IgG and C1q staining in the glomerular endothelium at 2 to 6 hours, with a clearly defined vascular loop structure. From 48 hours onwards, the C3 deposits lining the vascular endothelium become more prominent, and significant $\mathrm{C} 3$ staining is present in the lumen independent from rabbit IgG deposits (Figure 3a). With the same mice, we found clear colocalization of properdin and C3 throughout the observation period, suggestive of AP-mediated C3 activation (Figure 3b). However, the single deposits of both $\mathrm{C} 3$ and properdin were also observed, especially at 48 to 72 hours, when properdin staining was most prominent (Figure $3 b$ ). 
a

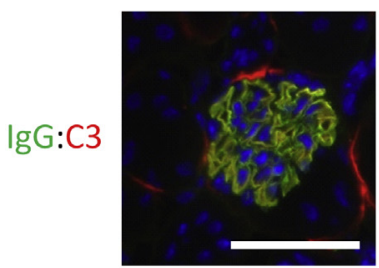

b

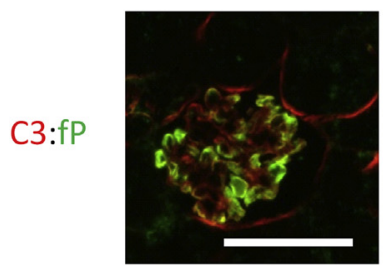

$6 \mathrm{~h}$

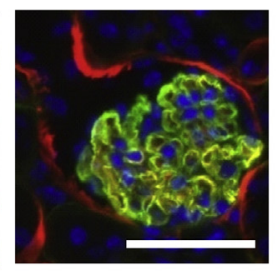

$6 \mathrm{~h}$

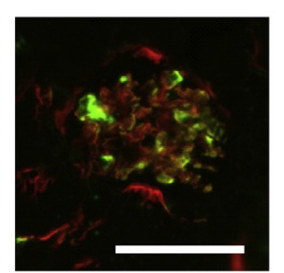

$24 \mathrm{~h}$

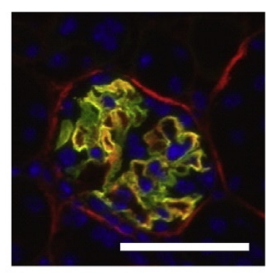

$24 \mathrm{~h}$

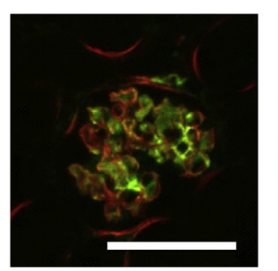

$48 \mathrm{~h}$

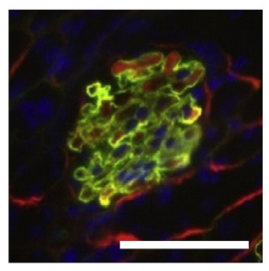

$48 \mathrm{~h}$

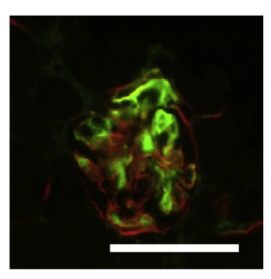

$72 \mathrm{~h}$

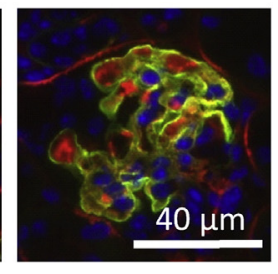

$72 \mathrm{~h}$

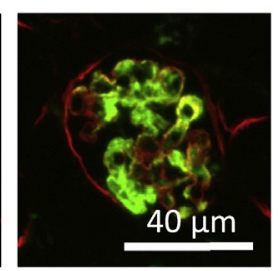

Figure 3 | Time-course colocalization of rabbit anti-glomerular basement membrane (GBM) IgG with mouse C3, and mouse C3 with properdin (fP). Analysis was performed with kidneys harvested from wild-type C57bl/6 mice killed 2 to 72 hours (h) after administration of antiGBM IgG. (a) Anti-GBM rabbit IgG (green) was detected with Alexa-488-labeled secondary antibody and C3 (red) with Alexa-568-labeled secondary antibody, and nuclei (blue) were stained with Hoechst. (b) Mouse properdin (green) was detected with HRP-labeled secondary antibody and tyramide FITC amplification, and C3 (red) with PE-labeled secondary antibody. Images were acquired at $40 \times$ original magnification, 4 mice ( 2 female and 2 male) per group were analyzed, and the representative glomerulus was chosen from images acquired from 4 different animals. To optimize viewing of this image, please see the online version of this article at www.kidney-international.org.

\section{Properdin deposition in injured glomeruli independent of C3 involvement}

Properdin deposition in $\mathrm{C} 3-\mathrm{KO}$ mice was demonstrated at various time points $(2,24$, and 72 hours) following anti-GBM antibody treatment (Figure 4a). Colocalization of rabbit IgG with properdin was only incidental, and properdin exhibited strong single staining in both WT and C3 KO mice, with no evident staining in $\mathrm{fP} \mathrm{KO}$ mice (Figure $4 \mathrm{~b}$ ). To investigate cell death, we performed terminal deoxynucleotidyl transferase dUTP nick-end labeling (TUNEL) staining in 72-hour biopsies (Figure 4c). TUNEL assay positivity was observed in glomeruli staining positive for rabbit IgG, C3, and properdin. However, when looking in detail, especially for C3 and properdin, only a minor fraction showed colocalization (Figure 4c). Analysis of TUNEL and properdin staining in C3 KO mice showed close but nonoverlapping colocalization within glomeruli at 72 hours, and properdin staining was more abundant compared with TUNEL staining (Figure $4 d$ ).

\section{Glomerular injury and proteinuria are not prevented in C3 and properdin KO mice}

Next, we evaluated the impact of properdin $\mathrm{KO}$ on the antiGBM antibody-induced renal injury using urine albumincreatinine ratio (UACR) as a functional read-out (Figure 5). The injection of anti-GBM antibody in WT C57bl/6 resulted in severe albuminuria in 24-hour urine collected between 72 and 96 hours. The UACR in WT mice was significantly higher in anti-GBM antibody-injected mice compared with control antibody-injected mice $(60,761 \pm 30,011 \mathrm{mg} / \mathrm{g}$ and $1205 \pm$ $282 \mathrm{mg} / \mathrm{g}$, respectively). Compared with WT mice, the properdin $\mathrm{KO}$ and C3 KO mice showed a 36\% to $40 \%$ lower level of albuminuria (UACR of $36,420 \pm 16,994 \mathrm{mg} / \mathrm{g}$ and $40,373 \pm 18247 \mathrm{mg} / \mathrm{g}$, respectively). However, statistical difference was not reached. Female WT and C3 KO mice exhibited a higher degree of renal injury than did male mice. In properdin $\mathrm{KO}$ mice no gender differences were observed (Supplementary Table S1).

\section{Neutrophil activation and inflammation after administration of anti-GBM antibody}

Neutrophils have been shown to contribute to renal injury in experimental models of anti-GBM disease. ${ }^{24}$ Time-course analysis of neutrophils using a GR1 staining showed that infiltration within the glomeruli was biphasic, being most prominent at 2 hours, returning to low levels at 24 to 48 hours, and increasing at 72 hours (Figure 6a). Acute infiltration and activation of neutrophils was supported by a transient 3-fold increase in serum myeloperxodase (MPO) at 2 to 6 hours (Figure 6b), which was followed with serum amyloid protein (SAP) peaking at 24 hours and remaining elevated until 72 hours (Figure 6c). Serum properdin showed a gradual increase from baseline, which reached significance at 48 hours (Figure 6d).

\section{Dependence of neutrophil infiltration on properdin}

Complement anaphylatoxins $\mathrm{C} 3 \mathrm{a}$ and $\mathrm{C} 5 \mathrm{a}$ are potent chemoattractants for inflammatory cells. Additionally, neutrophils contribute to the systemic properdin pool and can secrete it locally. ${ }^{25,26}$ There was no clear colocalization with properdin and GR1-positive neutrophils in the affected glomeruli at 2 hours, and neither properdin nor neutrophils were present in control antibody-treated mice (Figure 7a). Furthermore, the degree of neutrophil infiltration was not decreased in properdin $\mathrm{KO}$ mice (Figure $7 \mathrm{~b}$ ), suggesting that in this model properdin-regulated activation of complement does not contribute to recruitment of neutrophils. 
a

fP

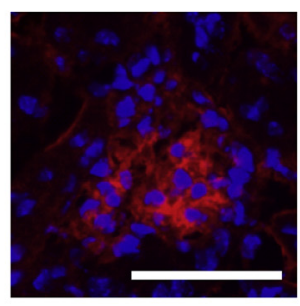

b

C

UNEL

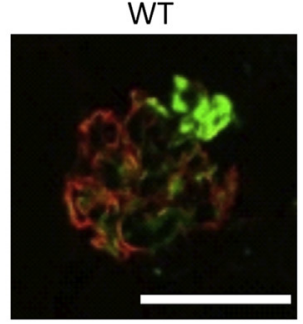

$\lg G$

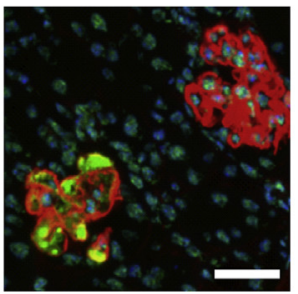

d

$\mathrm{C} 3 \mathrm{KO}$

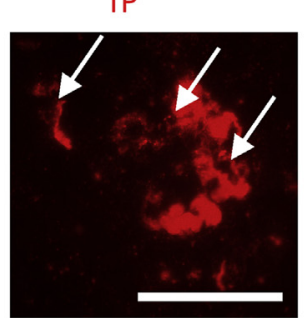

$24 \mathrm{~h}$

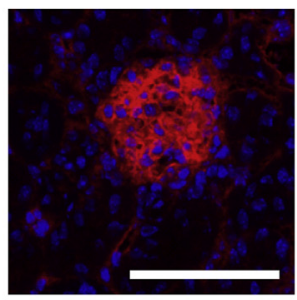

C3 KO

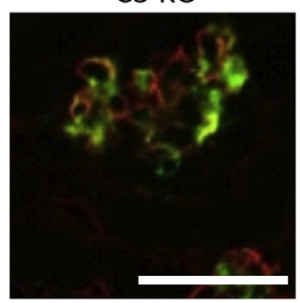

C3

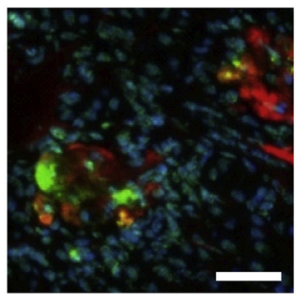

Merge

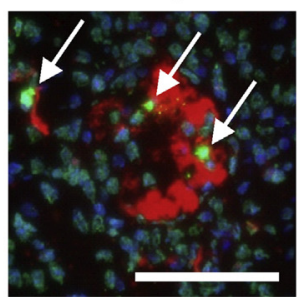

$72 \mathrm{~h}$

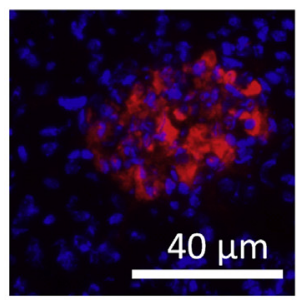

fP KO

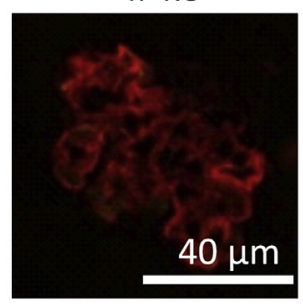

fP

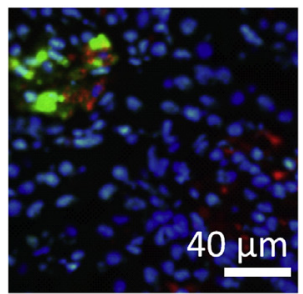

TUNEL

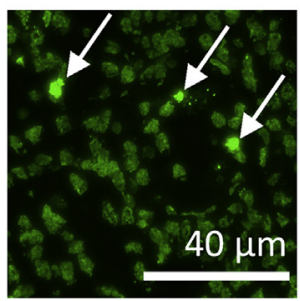

Figure 4 | Characterization of properdin binding to injured glomeruli. (a) Time course of properdin (fP) deposition (red) in C3 knockout (KO) mice treated with rabbit anti-GBM at 2, 24, and 72 hours (h) was detected with PE-labeled secondary antibody and counterstained with Hoechst. (b) Localization of fP (green) stained with tyramide-FITC amplification in relation to disease-inducing rabbit IgG (red) stained with PE-labeled secondary antibody in wild-type (WT), C3 KO, and fP KO mice at 72 hours after anti-GBM antibody administration. (c) Colocalization of terminal deoxynucleotidyl transferase dUTP nick-end labeling (TUNEL)-stained cells (green) with IgG, C3, and fP deposition detected with PE-labeled secondary antibody (red) in WT mice at 72 hours. Cells are stained with Hoechst (blue). (d) Colocalization of fP with TUNEL-positive cells at 72 hours in C3 KO mice treated with anti-GBM lgG. Arrows indicate TUNEL-positive cells and their location in the overlay images. To optimize viewing of this image, please see the online version of this article at www.kidney-international.org.

\section{DISCUSSION}

Recent studies have established that properdin has differing roles in disease pathologies, with a contributing role in experimental models of renal IRI and possibly arthritis and a protective role in C3 glomerulopathy and sepsis. ${ }^{18,20,27,28}$ The role of properdin as a positive regulator of AP is clear. However, the possible PRM role remains unsolved. Recent in vitro studies show that properdin has PRM activity and can act as a focus point of local C3b deposition on several ligands. ${ }^{8,9,13,15,29}$ Conversely, in other cases properdin has been shown to act only as a positive regulator, requiring preceding C3b deposition to bind. $^{30,31}$ To better understand the mechanism of properdin binding and function in vivo, we explored an experimental model of anti-GBM disease in WT, properdin $\mathrm{KO}$, and $\mathrm{C} 3 \mathrm{KO}$ mice.

Our model exhibited typical characteristics of an experimental anti-GBM disease, which include systemic complement consumption, activation of AP and CP pathways, deposition of complement factors in the glomeruli, neutrophil activation, inflammation, and severe renal injury. ${ }^{23,24,32-34}$ Acute complement activation was verified with plasma $\mathrm{C} 3 \mathrm{~b} / \mathrm{C} 3 \mathrm{c} / \mathrm{iC} 3 \mathrm{~b}$ fragments at 2 hours, which also revealed the impact of properdin as a positive regulator of AP because properdin $\mathrm{KO}$ mice had a lower basal level of C3 activation fragments and less prominent generation of C3 fragments. Furthermore, we observed acute consumption of $\mathrm{CP}$ and $\mathrm{AP}$, which was not 


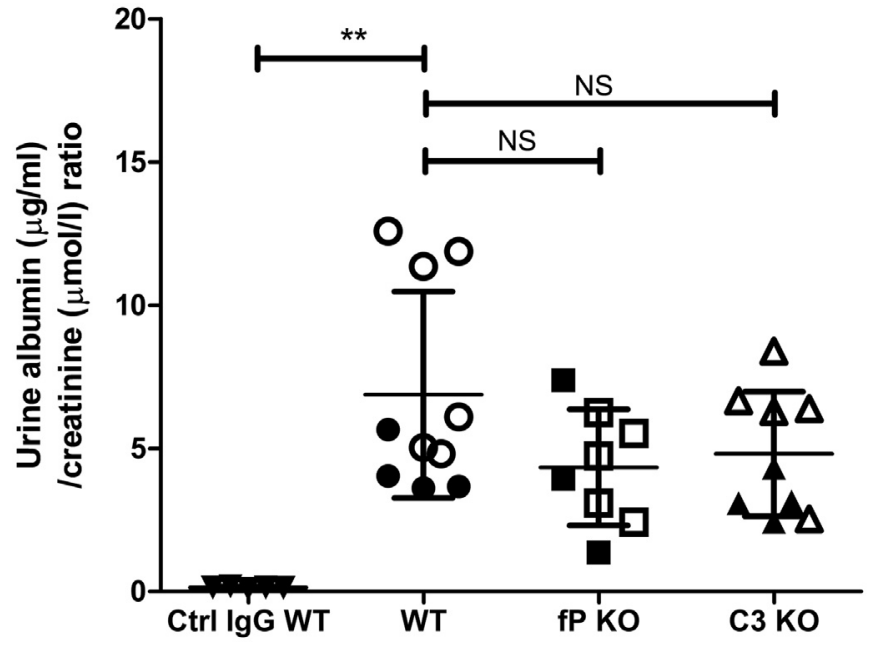

Figure 5 | Anti-glomerular basement membrane (GBM) diseaseinduced proteinuria. Anti-GBM disease was induced with injection of $0.5 \mathrm{mg}$ of rabbit anti-GBM lgG in wild-type (WT) C57bl/6 mice $(n=10)$, properdin (fP) knockout (KO) mice $(n=8)$, and C3 KO mice $(n=9)$. The control group WT $\mathrm{C} 57 \mathrm{bl} / 6$ mice were injected with $0.5 \mathrm{mg}$ of control rabbit lgG (Ctrl lgG WT; $n=5)$. The sex of each mouse is indicated in the figure by either solid (male) or outlined (female) symbols. Twenty-fourhour urine was collected from each mouse in metabolic cages at 72 to 96 hours for quantification of urinary albumin-to-creatinine ratio.

Nonparametric 1-way analysis of variance with Dunn's multiple comparison test was used to determine statistical significance. Error bars indicate SD. ${ }^{* *} P<0.01$. NS, not significant.

present in properdin $\mathrm{KO}$ mice, underlining the role of the properdin and AP amplification loop. ${ }^{5}$ Interestingly, we did not observe serum properdin consumption in WT mice. Conversely, the levels increased in tandem with SAP, suggesting that systemic inflammation may affect the secretion of properdin to circulation from activated inflammatory cells..$^{25,35,36}$ It must be noted that the range of mouse properdin seemed high compared with concentrations in human serum. This may be due to the use of recombinant mouse properdin as a standard that may not have the same distribution of properdin isoforms as is found in vivo. ${ }^{37}$

Time-course immunohistochemistry revealed an acute C1q, C3, and properdin deposition in line with serum determinations, suggesting CP- and AP-mediated complement activation. However, C6 and C9 deposition became evident only after 24 hours and continued to intensify from 48 to 72 hours, coinciding with increased C3 and properdin deposition. Together, these results suggest that in this model of antiGBM disease, the complement-mediated injury may be attenuated until 48 to 72 hours. Further analysis of C3 colocalization confirmed that initial complement activation is most likely CP dependent and is present along the vascular endothelium. However, after 48 hours the C3 staining intensified and colocalized more with properdin, suggesting $\mathrm{AP}$ and properdin involvement in complement activation. We demonstrated that properdin deposition was present in glomeruli of anti-GBM-injected C3 KO mice. We showed some colocalization of properdin with TUNEL-positive cells, potentially confirming interaction with apoptotic cells. ${ }^{12,38}$
However, a large part of the properdin was independent of TUNEL, thereby indicating the presence of other, yet unknown ligands.

Properdin $\mathrm{KO}$ and $\mathrm{C} 3 \mathrm{KO}$ resulted in reduced proteinuria compared with the WT mice; however, this protection was not statistically significant. This is in contrast to previous studies with a 2-step model, which established involvement of both complement and neutrophils in the experimental renal injury in male mice. ${ }^{24}$ Although small group size did not allow for proper statistical analysis, female mice seemed to exhibit markedly higher proteinuria in WT and $\mathrm{C} 3 \mathrm{KO}$ mice in this model. We did not observe differences in complement stains between female and male mice, including C6 and C9. We have recently shown that female $\mathrm{C} 57 \mathrm{bl} / 6$ mice have significantly reduced terminal complement pathway functionality. ${ }^{39}$ In light of our results here, we can conclude that this residual complement activity results in prominent complement deposition in vivo when the injury is localized, such as in the glomeruli. Our results show an acute and transient infiltration of neutrophils, with simultaneous increase of serum MPO and SAP that precedes the full complement activation. Furthermore, properdin KO did not impact neutrophil infiltration, and no colocalization between neutrophils and properdin was observed. Together our results show that in this model the initial CP- and AP-mediated $\mathrm{C} 3$ activation and anti-GBM IgG are sufficient to recruit neutrophils to the glomeruli together with the rabbit IgG Fc receptor. Our results show only limited amelioration of renal injury using C3 $\mathrm{KO}$ mice, suggesting that the neutrophils are the likely driver of the acute injury, and that the full complement activation dependent on $\mathrm{CP}, \mathrm{AP}$, and properdin is attenuated and may become a contributing factor later. ${ }^{24,40}$

Taken together, our results show that properdin is present in injured glomeruli in vivo, independent of C3 involvement. The mechanism and putative ligand facilitating the deposition remains unclear, as only incidental colocalization was found with C3, TUNEL-positive cells, neutrophils, and Igs. Further studies should aim to identify whether this finding is a result of specific epitopes or passive entrapment within the injured glomeruli, and whether deposition of properdin may direct further $\mathrm{C} 3$ activation in vivo. These findings are interesting for various AP- and complement-mediated diseases. The ability of properdin to interact independent of C3 in vivo expands its possible roles in diseases and injuries. In particular, the role of properdin in removal of apoptotic material ${ }^{12,16}$ and in neutrophil-mediated diseases ${ }^{29,41-43}$ can be expanded further with future in vivo studies using the methods, animals, and reagents described here.

\section{METHODS}

\section{Study animals}

C57BL/6 WT mice were purchased from Charles River Laboratories (Wilmington, MA). C3 KO mice were a kind gift from Mike Carroll (Harvard Medical School, Boston, MA), and properdin KO mice were maintained by Cordula Stover (University of Leicester, Leicester, UK). ${ }^{28}$ All experiments were approved by the Leiden 
a
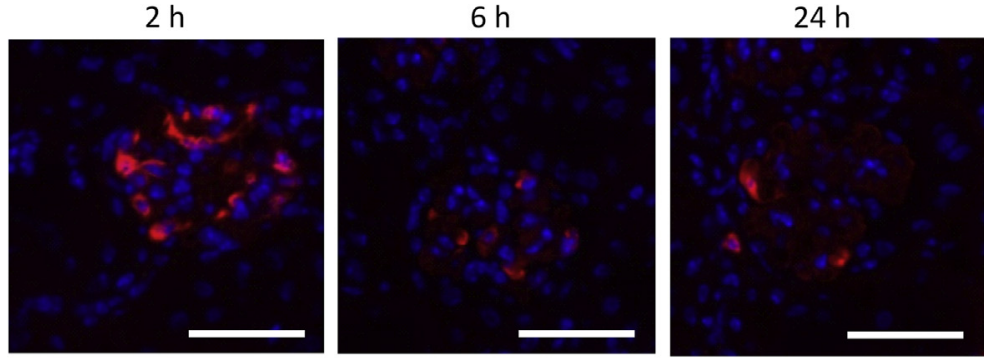

b

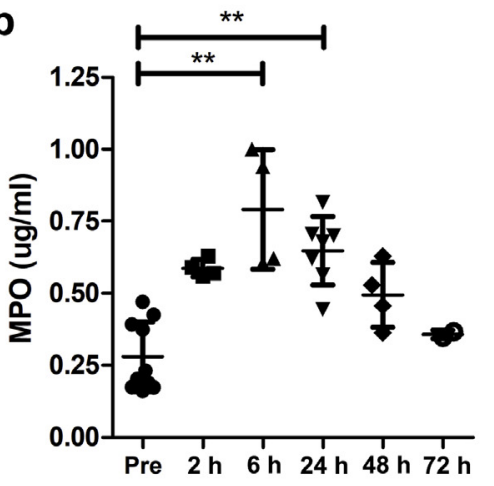

WT mice (hours post-injection)
C

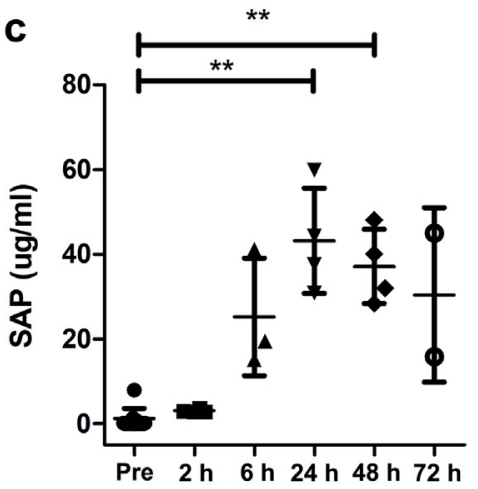

WT mice (hours post-injection)
$48 \mathrm{~h}$

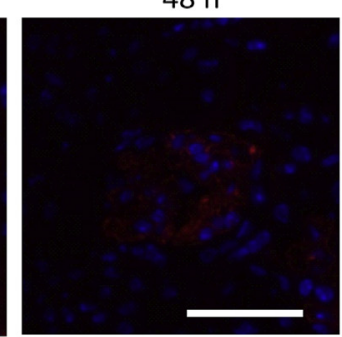

$72 \mathrm{~h}$

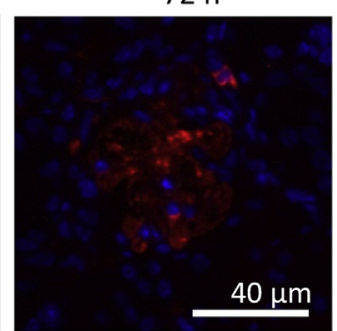

Figure 6 | Analysis of neutrophil activation in a 1-step model of anti-glomerular basement membrane (GBM) disease. (a) Wild-type (WT) mice were injected with anti-GBM IgG, and tissues were collected at different time points for staining of infiltrating neutrophils (red) with Alexa-568-labeled secondary antibody and rat anti-mouse GR1, and overlaid with Hoechst nuclear stain (blue). Activation of neutrophils was further analyzed with (b) serum myeloperxodase (MPO) measurement, and the impact on general inflammation with (c) serum amyloid protein (SAP). (d) Finally, the impact of anti-GBM IgG to complement was assessed with time-course determination of serum properdin. All measurements were done in at least duplicate, and statistical significance was determined with 1-way analysis of variance. ${ }^{*} P<0.05,{ }^{* *} P<0.01$. To optimize viewing of this image, please see the online version of this article at www.kidney-international.org.

University animal ethical committee and performed according to institutional and national guidelines.

\section{Induction of glomerulonephritis}

Rabbit anti-rat GBM IgG, cross-reacting with mouse GBM, was prepared in house as previously described, ${ }^{44}$ and preparation and cross-reactivity were confirmed in pilot experiments with anti-GBM and control antibodies injected into WT C57BL/6 mice. For the model, both male and female age-matched (range: 7-10 weeks old) mice (C57BL/6 WT, C3-KO, and properdin KO mice) were used. At day 0 , the mice were injected i.v. with rabbit anti-GBM or control rabbit IgG ( $0.5 \mathrm{mg}$ in $200 \mu \mathrm{l}$ physiological saline).

Acute anti-GBM disease was evaluated at 2 hours after injection. WT mice were injected with anti-GBM or control antibody, whereas properdin $\mathrm{KO}$ mice were injected only with anti-GBM antibody. For complement determinations, ethylenediamine tetra-acetic acid plasma was collected by tail cuts from each mouse before and 2 hours after injection without anesthesia. Serum was collected after $\mathrm{CO}_{2}$ killing via heart puncture. All blood samples were placed directly on ice and prepared as plasma or serum as described previously. ${ }^{45}$

The impact of $\mathrm{C} 3$ and properdin deficiency was investigated by injecting either anti-GBM or control $\operatorname{IgG}(0.5 \mathrm{mg})$ in WT, C3 KO, and properdin $\mathrm{KO}$ mice. The severity of renal injury was evaluated by 24-hour urine collection and proteinuria analysis from anti-GBM antibody-injected WT $(n=10)$, properdin-KO $(n=8)$, and C3 $\mathrm{KO}(n=9)$ mice and WT mice injected with control antibody $(n=5)$. For urine collection mice were placed in metabolic cages 72 hours after anti-GBM antibody administration with free access to food and water. The collected urine was centrifuged, aliquoted, and stored at $-20^{\circ} \mathrm{C}$.

The kinetics of complement and neutrophil activation were studied with groups of WT mice killed at 2 to 72 hours after injection of anti-GBM IgG. Additional kidneys were collected at 2 hours from properdin $\mathrm{KO}$ mice, and at 72 hours from $\mathrm{C} 3 \mathrm{KO}$ mice that were injected with either anti-GBM or control IgG. The mice were killed with $\mathrm{CO}_{2}$, and kidneys were harvested and snap-frozen for later histological analysis.

\section{Measurement of creatinine and proteinuria}

Urine albumin was quantified using rocket immunoelectrophoresis as previously described. ${ }^{24}$ Urine creatinine was quantified with creatinine strips for the Reflotron Plus system (Roche Diagnostics, Risch-Rotkreuz, Switzerland). The degree of proteinuria was calculated as the UACR.

\section{Properdin ELISA}

A specific ELISA for mouse properdin was developed with mouse anti-mouse properdin monoclonal antibody (clone 17-17) and rabbit polyclonal antibody raised against recombinant mouse properdin. ${ }^{46}$ The capture monoclonal antibody was coated at 2 $\mu \mathrm{g} / \mathrm{ml}$ in 96-well ELISA plates (Nunc Maxisorp; Thermo Fisher Scientific, Waltham, MA). Following coating, the wells were blocked with $1 \%$ bovine serum albumin in phosphate buffered saline (PBS). Normal mouse CD-1 serum, recombinant mouse properdin, or properdin $\mathrm{KO}$ serum was serially diluted in PBS, 
a
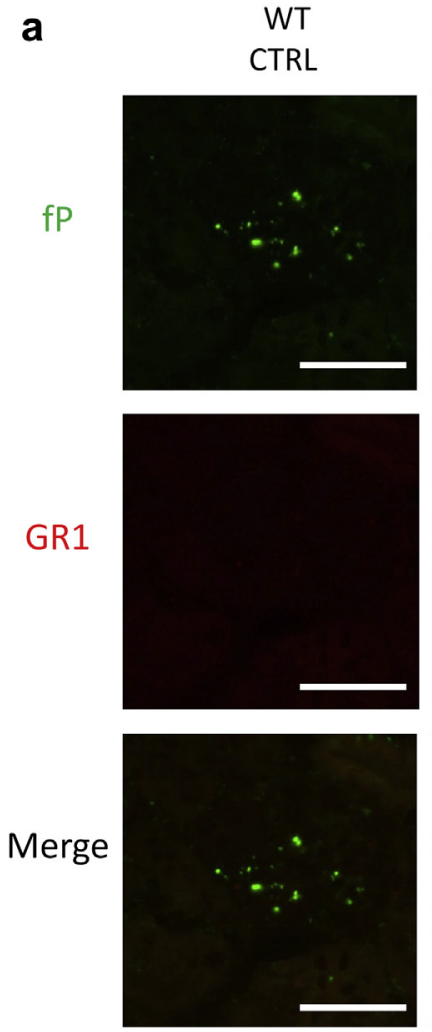

WT Anti-GBM
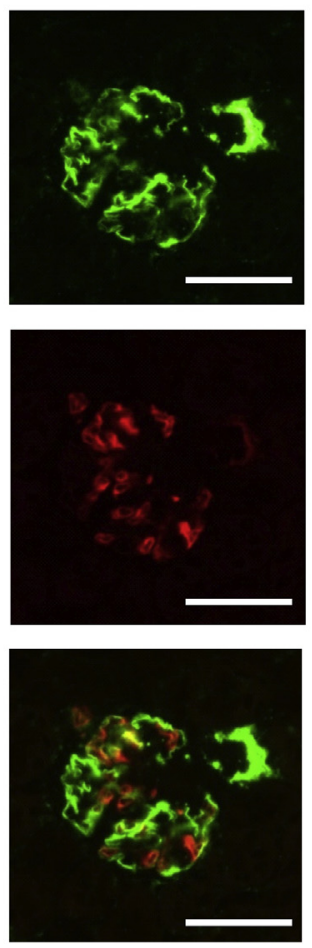

fP KO

Anti-GBM
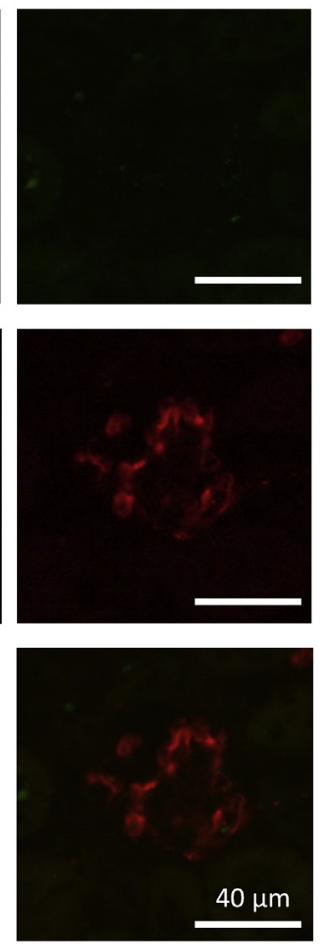

b

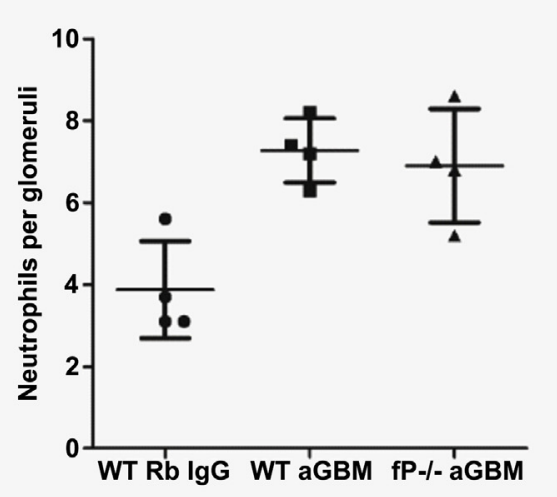

Figure 7 | Colocalization of neutrophil infiltration with properdin deposition in anti-glomerular basement membrane (GBM) antibodyaffected glomeruli. (a) The double staining of properdin and neutrophils (GR1) was performed 2 hours after anti-GBM IgG administration. GR1 was detected with Alexa-568-labeled secondary antibody (red) and properdin with tyramide-FITC amplification (green). Deposition was evaluated in wild-type (WT) mice injected with control antibody, WT mice injected with anti-GBM antibody, and properdin knockout (KO) mice injected with anti-GBM antibody. (b) Based on the GR1 stain the neutrophils were quantified and calculated as infiltrating cells per glomeruli. Rb IgG, rabbit anti-GBM IgG. To optimize viewing of this image, please see the online version of this article at www.kidney-international.org.

0.05\% Tween 20 (Sigma-Aldrich, St. Louis, MO), 1\% bovine serum albumin (phenacyl thiazolium bromide) and added to the blocked wells. Captured properdin was detected using digoxigenin (DIG)-labeled rabbit anti-human properdin (in house, Leiden University Medical Center), anti-DIG-POD (11207733910, Roche Diagnostics), followed by colorimetric quantification with a colorimetric substrate step with 3,3',5,5'-tetramethylbenzidine for 15 to 30 minutes at room temperature and stopped with $50 \mu \mathrm{l} 1 \mathrm{M}$ $\mathrm{H}_{2} \mathrm{SO}_{4}$ and read at $450 \mathrm{~nm}$ with a Bio-Rad 550 instrument (BioRad, Hercules, CA).

\section{Complement activation fragments and functional complement analysis}

Measurement of functional mouse complement pathway activities was performed with an ELISA-based system as described previously. ${ }^{39,45}$ In brief, human IgM-coated plates were used for CP, Mannan-coated plates for LP, and LPS-coated plates for AP ELISA. For $\mathrm{CP}$ and $\mathrm{LP}$ assessment plasma samples were diluted in $\mathrm{MgCl}_{2^{-}}$ and $\mathrm{CaCl}_{2}$-supplemented Veronal buffered saline, and for AP samples Veronal buffer supplemented with $\mathrm{MgCl}_{2}$ and $\mathrm{Ca}^{2+}$-chelating EGTA. Complement activity at the level of $\mathrm{C} 3$ was determined with biotinylated rat anti-mouse $\mathrm{C} 3 \mathrm{~b}-\mathrm{C} 3 \mathrm{c}-\mathrm{iC} 3 \mathrm{~b}$ monoclonal antibody clone 2/11 ${ }^{47}$ (HM1065; Hycult Biotech, Uden, Netherlands) and Streptavidin-HRP conjugate (Hycult Biotech).

\section{Determination of activated mouse C3, MPO, and SAP}

Commercial assays were used to measure SAP (HK215, Hycult Biotech) and MPO (HK210, Hycult Biotech) according to manufacturer's specifications. The $\mathrm{C} 3 \mathrm{~b} / \mathrm{C} 3 \mathrm{c} / \mathrm{iC} 3 \mathrm{~b}$ quantification was performed as described previously. ${ }^{45}$

\section{Histological analysis}

Kidneys were sectioned into $4-\mu \mathrm{m}$ slices using a cryostat, fixed by 10 minute incubation in acetone, and washed with PBS 3 times $5 \mathrm{mi}-$ nutes after each step, and all antibodies were diluted in $1 \%$ bovine serum albumin and PBS.

Mouse properdin, C6, and C9 were detected using tyramidefluorescein isothiocyanate (FITC) amplification-based staining. The slides were first treated with 45-minute room temperature incubation in PBS buffer containing $0.6 \% \mathrm{H}_{2} \mathrm{O}_{2}$ (1.07209.0250; Merck KGaA, Darmstadt, Germany) and $0.2 \% \mathrm{NaN}_{3}$ (1.06688.0100, Merck) diluted in PBS.

Properdin was detected with $1 \mu \mathrm{g} / \mathrm{ml}$ diluted rabbit anti-mouse properdin-DIG described for the properdin ELISA (in house, Leiden University Medical Center), whereas mouse C6 and C9 were detected with $1 \mu \mathrm{g} / \mathrm{ml}$ rabbit anti-mouse recombinant C6-DIG and C9-DIG described previously. ${ }^{45}$ For both components, the detection was followed with overnight incubation of the slides with 1/750 diluted sheep anti-DIG-POD (Roche Diagnostics). Finally, the slides 
were incubated 20 minutes with $1 / 500$ diluted tyramide-FITC (T9034-4, Sigma-Aldrich) in tyramide buffer (NENTM, Life Science Products, Chestertown, MD) with $0.01 \% \mathrm{H}_{2} \mathrm{O}_{2}$ (Merck).

Mouse GR1 was detected with 1/400 diluted rat anti-mouse GR1 (a kind gift from Georg Kraal, VU University Medical Center, Amsterdam, Netherlands) and 1/750 diluted goat anti-rat-Alexa 568 (A11011; Molecular Probes, Eugene, OR). Deposition of rabbit IgG to mouse glomeruli was detected with $1 / 400$ diluted goat anti-rabbit IgG-Alexa 488 (A11008, Molecular Probes) or 1/400 diluted goat anti-rabbit IgG-Alexa568 (ab175471; Abcam, Cambridge, UK). Mouse C3 was detected with 1/1000 rat anti-mouse C3 (CL75603AP; Cedarlane, Burlington, Canada), and 1/750 diluted goat anti-rat Alexa 568 (A11011, Molecular Probes). Where applicable, nuclear stains were performed with 1/10000 diluted Hoechst (H3569, Invitrogen, Carlsbad, CA) according to the manufacturer's instructions. Fluorescence microscopy was performed with a Zeiss Axio Scope A1 with an EX-plan Neofluar 40x lens and captured with Axiocam MRC5 (Carl Zeiss, Dublin, CA).

Specificity of the staining was confirmed with renal tissue harvested 72 hours after injection of antibody. Rabbit IgG and C9 stain specificity was controlled with mice injected with control rabbit IgG. Properdin and $\mathrm{C} 3$ staining specificity was tested with properdin $\mathrm{KO}$ and $\mathrm{C} 3 \mathrm{KO}$ mice injected with anti-GBM antibody. In all cases the staining was free of nonspecific signal within the glomeruli, as assessed by isotype controls.

\section{DISCLOSURE}

All the authors declared no competing interests.

\section{ACKNOWLEDGMENTS}

This work was financially supported in part by the European Union (Marie Curie TranSVIR FP7-PEOPLE-ITN-2008 no. 238756) and by a Consortium grant from the Dutch Kidney Foundation (COMBAT, the Netherlands).

\section{SUPPLEMENTARY MATERIAL}

Table S1. Analysis of proteinuria and impact of gender to renal injury. Table depicts the urinary albumin-creatinine ratio (UACR), urinary albumin $(\mu \mathrm{g} / \mathrm{ml})$ and creatinine $(\mu \mathrm{mol} / \mathrm{l})$. The 24 -hour urine was collected between 72 to 96 hours in individual metabolic cages from wild-type C57bl/6 (WT), properdin (fP) knockout (KO), and C3 KO mice. Values are depicted as mean \pm SD.

Figure S1. Specificity of renal histology stains. (A) The specificity of properdin (fP) and C3 stains were assessed with fP knockout (KO) and C3 KO mice, respectively, and compared against wild-type (WT) mice. The mice were injected with $0.5 \mathrm{mg}$ rabbit $(\mathrm{Rb}) \mathrm{lg}$ anti-glomerular basement membrane (GBM) antibody, and tissues were harvested at 72 hours. (B) Specificity of Rb lgG, C9, and GR1 stains were evaluated against tissues collected from WT mice injected with either $0.5 \mathrm{mg}$ nonspecific Rb lgG or 0.5 mg Rb lgG anti-GBM. Mouse C9 assessment was performed on tissues collected at 96 hours after injection, whereas GR1 and Rb lgG were assessed with tissues collected at 2 hours after injection.

Supplementary material is linked to the online version of the paper at www.kidney-international.org.

\section{REFERENCES}

1. Walport MJ. Complement. First of two parts. N Engl J Med. 2001;344: 1058-1066.

2. Camous L, Roumenina L, Bigot S, et al. Complement alternative pathway acts as a positive feedback amplification of neutrophil activation. Blood. 2011;117:1340-1349.
3. Harboe M, Ulvund G, Vien L, et al. The quantitative role of alternative pathway amplification in classical pathway induced terminal complement activation. Clin Exp Immunol. 2004;138:439-446.

4. Kim DD, Song WC. Membrane complement regulatory proteins. Clin Immunol. 2006;118:127-136.

5. Lutz HU, Jelezarova E. Complement amplification revisited. Mol Immunol. 2006;43:2-12.

6. Fearon DT, Austen KF. Properdin: binding to C3b and stabilization of the C3b-dependent C3 convertase. J Exp Med. 1975;142:856-863.

7. Pillemer L, Blum L, Lepow I, et al. The properdin system and immunity. I. Demonstration and isolation of a new serum protein, properdin, and its role in immune phenomena. Science. 1954;120:279-285.

8. Kemper C, Atkinson JP, Hourcade DE. Properdin: emerging roles of a pattern-recognition molecule. Annu Rev Immunol. 2010;28:131-155.

9. Spitzer D, Mitchell LM, Atkinson JP, Hourcade DE. Properdin can initiate complement activation by binding specific target surfaces and providing a platform for de novo convertase assembly. J Immunol. 2007;179:26002608.

10. Klop B, van der Pol P, van Bruggen R, et al. Differential complement activation pathways promote $\mathrm{C} 3 \mathrm{~b}$ deposition on native and acetylated LDL thereby inducing lipoprotein binding to the complement receptor 1 . J Biol Chem. 2014;289:35421-35430.

11. O'Flynn J, LUMC. Properdin-dependent activation and control of immunehomeostasis and autoimmunity. Available at: https://openaccess. leidenuniv.nl/handle/1887/28275. Accessed December 17, 2014.

12. $\mathrm{Xu} \mathrm{W}$, Berger SP, Trouw $L$ a, et al. Properdin binds to late apoptotic and necrotic cells independently of $\mathrm{C} 3 \mathrm{~b}$ and regulates alternative pathway complement activation. J Immunol. 2008;180:7613-7621.

13. Gaarkeuken H, Siezenga MA, Zuidwijk K, et al. Complement activation by tubular cells is mediated by properdin binding. Am J Physiol Renal Physiol. 2008;295:F1397-F1403.

14. Zaferani $A$, Vivès $R R$, van der Pol $P$, et al. Identification of tubular heparan sulfate as a docking platform for the alternative complement component properdin in proteinuric renal disease. J Biol Chem. 2011;286: 5359-5367.

15. Zaferani $A$, Vivès RR, van der Pol $P$, et al. Factor $h$ and properdin recognize different epitopes on renal tubular epithelial heparan sulfate. J Biol Chem. 2012;287:31471-31481.

16. Kemper C, Mitchell LM, Zhang L, Hourcade DE. The complement protein properdin binds apoptotic T cells and promotes complement activation and phagocytosis. Proc Natl Acad Sci U S A. 2008;105:9023-9028.

17. Pedersen DV, Roumenina L, Jensen RK, et al. Functional and structural insight into properdin control of complement alternative pathway amplification. EMBO J. 2017;36:1084-1099.

18. Miwa T, Sato S, Gullipalli D, et al. Blocking properdin, the alternative pathway, and anaphylatoxin receptors ameliorates renal ischemiareperfusion injury in decay-accelerating factor and CD59 doubleknockout mice. J Immunol. 2013;190:3552-3559.

19. Kimura Y, Zhou L, Miwa T, Song WC. Genetic and therapeutic targeting of properdin in mice prevents complement-mediated tissue injury. J Clin Invest. 2010;120:3545-3554.

20. Dimitrova $P$, Ivanovska N, Schwaeble $W$, et al. The role of properdin in murine zymosan-induced arthritis. Mol Immunol. 2010;47:1458-1466.

21. Zhou HH-F, Yan H, Stover CMC, et al. Antibody directs properdindependent activation of the complement alternative pathway in a mouse model of abdominal aortic aneurysm. Proc Natl Acad Sci U S A. 2012;109:E415-E422.

22. Ma R, Cui Z, Liao YH, Zhao MH. Complement activation contributes to the injury and outcome of kidney in human anti-glomerular basement membrane disease. J Clin Immunol. 2013;33:172-178.

23. Ma R, Cui Z, Hu S-Y, et al. The alternative pathway of complement activation may be involved in the renal damage of human antiglomerular basement membrane disease. PLoS One. 2014;9:e91250.

24. Otten M a, Groeneveld TWL, Flierman R, et al. Both complement and $\lg \mathrm{G} f \mathrm{c}$ receptors are required for development of attenuated antiglomerular basement membrane nephritis in mice. J Immunol. 2009;183:3980-3988.

25. Wirthmueller U, Dewald B, Thelen M, et al. Properdin, a positive regulator of complement activation, is released from secondary granules of stimulated peripheral blood neutrophils. J Immunol. 1997;158:4444-4451.

26. Bao L, Wang Y, Haas M, Quigg RJ. Distinct roles for C3a and C5a in complement-induced tubulointerstitial injury. Kidney Int. 2011;80: 524-534. 
27. Ruseva MM, Vernon $\mathrm{K}$ a, Lesher AM, et al. Loss of properdin exacerbates C3 glomerulopathy resulting from factor $\mathrm{H}$ deficiency. J Am Soc Nephrol. 2013;24:43-52.

28. Stover CM, Luckett JC, Echtenacher B, et al. Properdin plays a protective role in polymicrobial septic peritonitis. J Immunol. 2008;180:3313-3318.

29. O'Flynn J, Dixon KO, Faber Krol MC, Daha MR, Van Kooten C. Myeloperoxidase directs properdin-mediated complement activation. J Innate Immun. 2014;6:417-425.

30. Harboe M, Garred P, Lindstad JK, et al. The role of properdin in zymosanand Escherichia coli-induced complement activation. J Immunol. 2012;189:2606-2613.

31. Harboe M, Johnson C, Nymo S, et al. Properdin binding to complement activating surfaces depends on initial C3b deposition. Proc Natl Acad Sci U S A. 2017;114:E534-E539.

32. Sheerin NS, Springall T, Carroll MC, et al. Protection against antiglomerular basement membrane (GBM)-mediated nephritis in C3- and C4-deficient mice. Clin Exp Immunol. 1997;110:403-409.

33. Groggel GC, Adler S, Rennke HG, et al. Role of the terminal complement pathway in experimental membranous nephropathy in the rabbit. J Clin Invest. 1983;72:1948-1957.

34. Boyce NW, Holdsworth SR. Anti-glomerular basement membrane antibody-induced experimental glomerulonephritis: evidence for dosedependent, direct antibody and complement-induced, cell-independent injury. J Immunol. 1985;135:3918-3921.

35. Schwaeble W, Huemer HP, Möst J, et al. Expression of properdin in human monocytes. Eur J Biochem. 1994;219:759-764.

36. Dixon KO, O'Flynn J, Klar-Mohamad N, Daha MR, van Kooten C. Properdin and factor $\mathrm{H}$ production by human dendritic cells modulates their T-cell stimulatory capacity and is regulated by IFN- $\gamma$. Eur J Immunol. 2017;47: 470-480.

37. Stover CM, McDonald J, Byrne S, et al. Properdin levels in human sepsis. Front Immunol. 2015;6:24.
38. Banda NK, Takahashi K, Wood AK, et al. Pathogenic complement activation in collagen antibody-induced arthritis in mice requires amplification by the alternative pathway. J Immunol. 2007;179:4101-4109.

39. Kotimaa J, Klar-Mohammad N, Gueler F, et al. Sex matters: Systemic complement activity of female C57BL/6J and BALB/CJ mice is limited by serum terminal pathway components. Mol Immunol. 2016;76:13-21.

40. Coxon A, Cullere X, Knight S, et al. Fc $\gamma$ RIII Mediates Neutrophil Recruitment to Immune Complexes. Immunity. 2001;14:693-704.

41. Xiao $H$, Schreiber $A$, Heeringa $P$, et al. Alternative complement pathway in the pathogenesis of disease mediated by anti-neutrophil cytoplasmic autoantibodies. Am J Pathol. 2007;170:52-64.

42. Gou S-J, Yuan J, Chen M, et al. Circulating complement activation in patients with anti-neutrophil cytoplasmic antibody-associated vasculitis. Kidney Int. 2012;83:129-137.

43. Xing GQ, Chen M, Liu G, et al. Complement activation is involved in rena damage in human antineutrophil cytoplasmic autoantibody associated pauci-immune vasculitis. J Clin Immunol. 2009;29:282-291.

44. Joosten SA, van Dixhoorn MGA, Borrias MC, et al. Antibody response against perlecan and collagen types IV and $\mathrm{VI}$ in chronic renal allograft rejection in the rat. Am J Pathol. 2002;160:1301-1310.

45. Kotimaa JJP, van Werkhoven MBMB, O'Flynn J, et al. Functional assessment of mouse complement pathway activities and quantification of $\mathrm{C} 3 \mathrm{~b} / \mathrm{C} 3 \mathrm{c} / \mathrm{iC} 3 \mathrm{~b}$ in an experimental model of mouse renal ischaemia/ reperfusion injury. J Immunol Methods. 2015;419:25-34.

46. Ali YM, Hayat A, Saeed BM, et al. Low-dose recombinant properdin provides substantial protection against Streptococcus pneumoniae and Neisseria meningitidis infection. Proc Natl Acad Sci U S A. 2014;111:5301-5306.

47. Mastellos D, Prechl JO, László G, et al. Novel monoclonal antibodies against mouse C3 interfering with complement activation: Description of fine specificity and applications to various immunoassays. Mol Immunol. 2004;40:1213-1221. 